\title{
Nordiques
}

33 | 2017

La transition des villes nordiques : quelles innovations territoriales en périphérie?

\section{Marie-Pierre Richard, La citoyenneté locale en Suède}

Presses universitaires du Septentrion, 2016

\section{Yohann Aucante}

\section{(Q) OpenEdition Journals}

Édition électronique

URL : https://journals.openedition.org/nordiques/3429

DOI : $10.4000 /$ nordiques.3429

ISSN : 2777-8479

Éditeur :

Association Norden, Bibliothèque de Caen la mer

\section{Édition imprimée}

Date de publication : 1 mai 2017

Pagination : 143-146

ISBN : 978-2-9544654-9-4

ISSN : $1761-7677$

Référence électronique

Yohann Aucante, "Marie-Pierre Richard, La citoyenneté locale en Suède », Nordiques [En ligne], 33 | 2017, mis en ligne le 19 janvier 2022, consulté le 02 février 2022. URL : http://

journals.openedition.org/nordiques/3429; DOI : https://doi.org/10.4000/nordiques.3429

Ce document a été généré automatiquement le 2 février 2022

Nordiques 


\section{Marie-Pierre Richard, La citoyenneté locale en Suède}

Presses universitaires du Septentrion, 2016

Yohann Aucante

\section{RÉFÉRENCE}

Marie-Pierre Richard, La citoyenneté locale en Suède, Presses universitaires du Septentrion, 2016, 236 pages

1 Issu d'une thèse de doctorat à l'université de Lille, l'ouvrage de Marie-Pierre Richard est bienvenu à plusieurs titres. Ainsi que le rappelle l'auteure, il n'existe guère de littérature en français sur les politiques territoriales en Europe du Nord (Suède en l'occurrence) et sur leurs implications en termes de citoyenneté, qui est l'objet central de l'ouvrage. $\mathrm{M}^{\text {me }}$ Richard, ayant étudié et vécu en Suède, a elle-même une connaissance pratique de première main, ce qui aurait d'ailleurs pu contribuer à rendre le contenu plus vivant en faisant un usage un peu plus fréquent d'observations concrètes et vécues.

2 L'hypothèse centrale du livre est qu'il existe un espace spécifique pour la manifestation d'une citoyenneté «locale», qui serait liée pour partie aux traditions fortes d'organisation et d'expression politique, notamment dans les communes, mais aussi à l'accroissement d'une immigration - intra ou extra nordique ${ }^{1}$ - qui bénéficie d'une citoyenneté politique au plan local (droit de vote pour certaines élections, droits sociaux, etc.). Cette immigration est conséquente, puisqu'on estime ses effectifs à environ 1,5 million sur une population totale de 10 millions $^{2}$. Ce dernier aspect est sans doute ce qui fait à la fois l'actualité et l'originalité de l'ouvrage de $\mathrm{M}^{\mathrm{me}}$ Richard.

Sur le premier volet, celui des spécificités d'une "libre administration " territoriale nordique, et notamment sur ses rapports ambivalents avec la structuration d'un État providence, il y aurait beaucoup à dire. Force est de constater que l'argumentation est assez désordonnée, sur un sujet d'une éminente complexité et qui aurait mérité plus de 
rigueur à la fois historique, conceptuelle et méthodologique. Dans la première partie, l'auteure distille ainsi des informations éparses sur les processus qu'elle décrit comme de la « décentralisation » ou de la « libre administration », sur les fusions territoriales (ambivalentes de ce point de vue dans la mesure où elles visaient à la fois à renforcer le contrôle de l'État, la mise en œuvre de politiques sociales homogènes et à rationaliser l'économie et l'organisation locales). Il n'y a guère de repères historiques pour rappeler qu'avant de devenir des « communes providences » à partir des années 1960-1970, les municipalités étaient aussi les héritières d'un système ancien et rétrograde d'assistance aux pauvres, tout en ayant progressivement et inégalement impulsé des réformes au diapason de la montée en puissance d'un État social.

4 Comme souvent dans le domaine pourtant extrêmement restreint en France des études nordiques, on pourra déplorer que l'auteure soit passée à côté des seules recherches en français qui portent sur ces questions et qui sont le fait de l'auteur de ces lignes, lequel tient par ailleurs un séminaire de recherche ciblé sur la protection sociale et ses dimensions territoriales depuis 2006 à l'EHESS ${ }^{3}$. Au plan historique, on navigue un peu à vue sans toujours savoir à quelle période on se réfère et sans rappel suffisant des grandes évolutions du système suédois de démocratie locale et sociale ou des rationalités politiques qui les animent (chapitre 1 et 2). Ainsi passe-t-on extrêmement rapidement sur les modalités et les conséquences des fusions, sur les expérimentations fondamentales des "communes libres" dans les années 1980, et sur ce qu'elles impliquent en matière de citoyenneté, mais aussi sur les transformations profondes au moment de la crise suédoise du début des années 1990 (lois Ädel par exemple et transferts de nombreuses responsabilités aux communes dans un contexte économique très tendu). Le rapport ambivalent de la social-démocratie à l'État et à ses territoires aurait dû faire l'objet de quelques paragraphes, pour comprendre comment la fusion des municipalités a d'abord obéi à un projet de rationalisation des entités locales en tant que rouages de l'État providence. Malheureusement, un tel traitement elliptique n'est pas de nature à éclairer les lecteurs français sur la stratification territoriale complexe de ces systèmes. À moindres frais, quelques cartes ou tableaux (à l'image de celui p. 28) auraient été utiles au lecteur désireux de mieux suivre ces évolutions. Les concepts sont aussi distillés au compte-gouttes sans vraiment qu'on explicite leur usage ("démarchandisation», "gouvernance douce/multiniveaux»), même pour celui central - de citoyenneté, qui est multidimensionnel (définition très succincte p. 21). Il y a cependant des passages de "description» de la citoyenneté sociale locale, qui donnent à en voir les aspects concrets (exemple p. 46-47).

5 L'expression politique de la citoyenneté apparaît au chapitre 3. La question des élections et des partis politiques au niveau local n'est pas abordée, au prétexte d'une abondante littérature existante (ce qui est vrai, mais pas en français). On s'intéresse donc à la citoyenneté envisagée sous ce qui est appelé les « contre-pouvoirs ", mais qui correspond dans les faits aux ressources légales dont les citoyens peuvent se prévaloir. Le principe de " publicité » (offentlighetsprincipen), curieusement non traduit en français (p.56), aurait lui aussi mérité un peu d'histoire, tant il confère à la Suède certaines spécificités. L'auteure en donne quelques illustrations et elle aurait pu aisément relier ces dimensions à la thématique de la confiance sociale qui lui semble être essentielle en Suède.

6 Après une première partie ayant vocation à décrire un "cadre général », les deux suivantes portent sur les évolutions et défis de la citoyenneté au niveau local, mais 
aussi sur certaines restructurations territoriales dont on aurait pu penser que leur place était plutôt dans la première partie. Il en va ainsi de la régionalisation abordée au chapitre 5 et qui aurait été plus intelligible si les évolutions profondes des années 1980-1990 avaient été décrites, avec la redistribution des compétences qui en a découlé ${ }^{4}$ (or cette description est partiellement faite, mais plus loin). C'est aussi là que la nécessité d'une carte se fait particulièrement sentir. Mais la frustration du lecteur vient surtout de l'énumération très longue de mesures ou de constats d'actions à chaque paragraphe, sans que cela serve à soutenir un argumentaire de fond sur la citoyenneté. On apprend certes beaucoup de choses, mais on passe rapidement de l'Europe à l'environnement, puis à la privatisation, sans avoir le temps de traiter ces questions fondamentales. Parvenir ainsi à évoquer les restructurations de la santé en trois paragraphes relève de l'exploit (p.118)! La question de l'affaiblissement de la démocratie locale dans ce contexte de nouvelle gestion publique à marche forcée a suscité beaucoup de littérature qui aurait pu soutenir l'argumentaire de l'auteur, à l'image de quelques remarques en ce sens, p. 120, par exemple.

7 Car c'est bien là que se situe l'un des paradoxes suédois : une démocratie locale à première vue ouverte et dynamique, mais qui doit cohabiter avec des formes de gouvernance publique fortement contraintes et en constante évolution depuis trente ans. Le journaliste Magnus Falkehed avait déjà abordé ces thématiques dans un ouvrage remarqué en France, évidemment intitulé Le modèle suédois ${ }^{5}$. L'ouvrage cherche donc à aborder trop de choses en peu de pages, à l'image des "politiques sectorielles " (urbanisme, politiques sociales et éducation!) traitées en quinze pages. L'auteure est ainsi contrainte d'effleurer la vitrine des belles réalisations suédoises, par exemple en matière de villes durables (Hammarby sjöstad), sans avoir la possibilité effective d'une interprétation ou d'un recul critique. Les citoyens en particulier, censés être au cœur de l'étude, n'apparaissent qu'en pointillé dans le processus, et à un niveau très général. Il aurait été bien plus stimulant de se concentrer sur une ou deux politiques, voire une étude de cas, par exemple dans le domaine éducatif qui a connu des transformations très profondes. Dans ce cas de figure, les «citoyens » sont les élèves/étudiants et les parents, leurs associations et, bien entendu, les institutions d'éducation populaire qui n'ont droit qu'à un paragraphe alors qu'elles portent une longue tradition. La fin du chapitre sur les questions d'éducation civique et de stratification sociale montre pourtant la bonne direction.

8 Le dernier chapitre questionne les évolutions de la citoyenneté au prisme de l'immigration et des inégalités au plan territorial. C'est là un axe essentiel de transformation de la société et de la politique suédoises contemporaines. Ici encore, c'est l'impression de kaléidoscope qui l'emporte, sans que l'on puisse suivre une ligne qui rattache clairement à la citoyenneté. L'action des associations locales, les mobilisations, la participation, l'innovation et le multiculturalisme ne sont bien souvent qu'effleurés, et pourtant, nous avions ici un point d'entrée particulièrement intéressant pour un ouvrage de ce type : au moment où l'ouvrage était rédigé, le parti nationaliste des démocrates de Suède était déjà en nette progression, notamment au niveau local, ce qui se traduisait par des visions très contrastées et conflictuelles de la citoyenneté.

9 In fine, c'est un sentiment de frustration qui domine à la lecture de cet ouvrage, dont le sujet est passionnant, essentiel, et très peu traité. Des références pertinentes sont mobilisées dans la bibliographie, on fournit au lecteur un grand nombre d'informations 
intéressantes, mais il y a un manque d'organisation du propos et de développement d'une analyse argumentée, se rattachant aux théories existantes sur la citoyenneté et la démocratie locale. À vouloir étudier les questions territoriales sous trop de dimensions et dans un format assez ramassé pour une thèse (200 pages), il paraît difficile d'approfondir et la citoyenneté ne marche pas vraiment comme fil conducteur. S'il est intéressant de s'appuyer sur la " littérature grise » très fournie, un minimum d'enquête empirique auprès des acteurs - ou une étude de cas plus détaillée - aurait rendu l'ouvrage plus vivant et concret. Les lecteurs qui n'ont pas de familiarité avec ces questions en Suède y trouveront toutefois une bonne source d'informations, accessible et loin du jargon qui peut se développer dans ce domaine.

\section{NOTES}

1. La différence est importante, les ressortissants des pays voisins étant traditionnellement nombreux à résider en Suède sans nécessairement demander la nationalité. Dans le cadre du marché commun nordique, cette libre circulation est assortie de bonnes garanties d'intégration et d'un accès sans restriction au droit de vote local. Pour d'autres catégories de migrants, plus nombreuses ces dernières années, existent des conditions de durée de résidence et les conditions d'intégration ne sont pas équivalentes.

2. Précisons qu'une partie de cette immigration provient de l'aire nordique.

3. Pour une synthèse, voir Yohann Aucante, Les démocraties scandinaves. Des systèmes politiques exceptionnels ?, Paris, A. Colin, 2013

4. Notons au passage, p.108-109, que l'échelon administratif intermédiaire entre commune et région n'est pas le Landsting (qui est l'organe représentatif) mais le Län.

5. Magnus Falkehed, Le modèle suédois. Santé, services publics, environnement. Ce qui attend les Français, Paris, Payot, 2005. 